\title{
Increased risk of gastric cancer in workers with occupational dust exposure
}

\author{
Mo-Yeol Kang ${ }^{1}$, Jiyoun Jung ${ }^{1}$, Jung-Wan Koo ${ }^{1}$, Inah Kim² ${ }^{2}$, Hyoung-Ryoul Kim¹ , and Jun-Pyo Myong ${ }^{1}$
}

${ }^{1}$ Department of Occupational and Environmental Medicine, Seoul St. Mary's Hospital, College of Medicine, The Catholic University of Korea, Seoul; ${ }^{2}$ Department of Occupational and Environmental Medicine, Hanyang University College of Medicine, Seoul, Korea

Received: December 8, 2019 Revised: February 13, 2020 Accepted: April 6, 2020

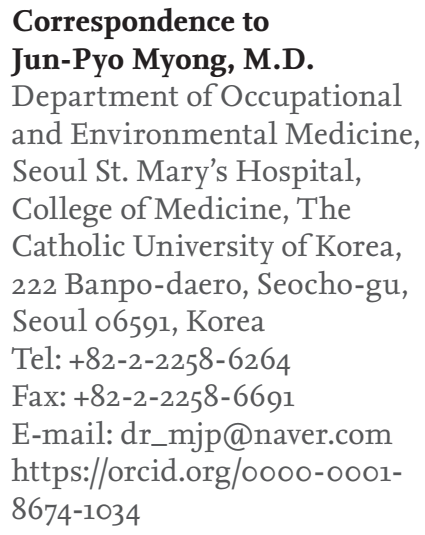

Background/Aims: Workers who are exposed to dust in the workplace tend to show a higher incidence of gastric cancer. Nevertheless, scientific evidence to support an association between dust exposure and the risk of gastric cancer is inadequate. This study aimed to investigate whether or not occupational dust exposure influences the risk of gastric cancer.

Methods: We collected the electronic data from the Pneumoconiosis Health Examination (PHE) program, provided by the Ministry of Employment and Labor and the Korea Workers' Compensation and Welfare Service from 2002 to 2017 . The PHE database was linked to the National Health Insurances databases. The age-standardized incidence ratio (SIR) and 95\% confidence intervals (CIs) of the risk of gastric cancers were evaluated in workers with occupational dust exposure, and the results were compared to those in the general population.

Results: From 2004 to 2015, 1,543 cases of gastric cancer were observed in the male participants, as compared with 1,174 of expected cases, which yielded an SIR of 1.314 (95\% CI, 1.249 to 1.380). Under dust exposure, the risk of gastric cancer was increased $23.9 \%$ in the male participants (95\% CI, 19.9 to 27.5), and the degree of impairment of lung function was inversely related to the risk of gastric cancer.

Conclusions: Workers with occupational dust exposure were at higher risk of developing gastric cancer than the general population. Thus, future efforts for the prevention of gastric cancer are necessary for dust exposed workers.

Keywords: Occupation dust; Stomach neoplasms; Incidence

\section{INTRODUCTION}

Miners, construction workers, and stonemasons are exposed to various types of dust, such as crystalline silica, in the workplace. Respirable dust that is inhaled may get deposited in the secondary bronchioles and alveolar area, and accumulate over time, which can lead to adverse health outcomes such as respiratory diseases including pneumoconiosis, chronic obstructive pulmonary disease, interstitial pulmonary fibrosis, and cancer (for example, lung cancer in exposure to crystalline sil- ica and diesel exhaust particles; nasopharyngeal cancer in exposure to metal dust and wood dust) [1-3].

Previous studies on the effects of environmental dust exposure on human health have mainly focused on the respiratory system [1-3]. Nevertheless, inhaled dust might migrate from the upper respiratory tract into the lower respiratory tract and can be swallowed and migrate into the digestive system, becoming a potential risk factor for organs. Despite limited knowledge of the relationship between dust exposure and cancer of the digestive tract, a meta-regression analysis on occupational exposure to 
crystalline silica suggests that development of digestive cancer may be related to previous exposure to dust in the work place [4].

Gastric cancer is the sixth most common cancer and second leading cause of cancer-related mortality worldwide [5]. It accounted for approximately 783,000 of cancer deaths in 2018 [6], and is mainly caused by infection with Helicobacter pylori bacteria [7]. Tobacco smoking and low socioeconomic status are related with moderate increase in the risk of gastric cancer [8]. In addition, dietary substances were considered as important risk factors [9].

Several studies have demonstrated that certain occupations increase the risk for gastric cancer [10,11]; particularly, dusty work environments and occupations that pre-expose the individual to carcinogens, are potential risk factors $[11,12]$. Despite these established facts, scientific evidence for an association between dust exposure and gastric cancer is still needed. One case-control study on the association between silica exposure and gastric cancer in Poland reported that the two phenomena may be related, but these results should be cautiously generalized due to limited number of participants in the study.

Mining was the main industrial activity in Korea for decades, but underwent decline in the late 1980s. Concomitantly, the number of workers in non-mining industries including stonemasonry, foundry, shipbuilding, and welding has been increasing. To address growing concern over long-term health effects of dust exposure, the Ministry of Employment and Labor and the Korea Workers' Compensation and Welfare Service (KCOMWEL) have developed Pneumoconiosis Health Examination (PHE) as a special health examination program for workers with occupational dust exposure. The main goal of PHE is screening and follow-up of the pneumoconiosis cases in Korea since 1984, to enable prevention of disease and protection of those affected. Moreover, National Health Insurance (NHI) is the official insurance in Korea and covers majority of the Korean population [13], and its databases provide relevant information of the users. Combined big data of dust exposure and health outcome from PHE and NHI would allow relevant epidemiologic studies. The present study aimed to investigate whether or not occupational dust exposure influences the risk for gastric cancer by using electronic data from PHE in combination with informa- tion obtained from the NHI databases.

\section{METHODS}

\section{Data source}

The electronic database of PHE provided by the Ministry of Employment and Labor and KCOMWEL from 2002 to 2017 was the main source for the present analysis. In each individual, information on work history, medical examination history, and the exposed dust type were collected. All chest X-rays were interpreted and classified using the International Labor Organization (ILO) scale by three radiologists who were well trained and experienced in evaluating pneumoconiosis.

The PHE database is linked to the databases of NHI, the mandatory social insurance program administered by the Korean government. NHI, Medical Aid, and longterm care insurance (LTCI) are the main healthcare programs that provide approximately $98 \%$ coverage in the Korean population [14], and the data of those programs are based on health insurance claims. Based on these facts, in the present study, the individual's health-related outcomes were assessed in all three databases.

According to the health insurance policies' guideline, all healthcare facilities in Korea submit the data of their medical services to the National Health Insurances Services (NHIS). The NHIS database includes qualification data and data of medical service: Qualification data comprise the age, sex, region, household income level, type of insurance, identification number, and family information, while medical service data comprise information of covered inpatient and outpatient visits, procedures, and prescriptions. All information on hospital visits in the Korea NHIS database were categorized using standard protocol of the Korea Classification of Diseases and Causes of Death 4 th edition (KCD), which corresponds to the International Classification of Diseases 1oth revision.

\section{Definition of variables}

In our study, the variables of age, chest radiography findings, and spirometry summary from the PHE database were submitted for analysis.

Examinees were classified into 10 groups according to age: less than 45,45 to 49,50 to 54,55 to 59,60 to 64 , 
65 to 69,70 to 74,75 to 79,80 to 84 , and $>84$ years (as of January 1st of each year). The radiography findings of pneumoconiosis were classified according to the profusion of small opacities as follows: Category o (no opacity); Category o/1 (suspected opacity), Category 1 (1/o, $1 / 1,1 / 2)$, Category $2(2 / 1,2 / 2,2 / 3)$, Category $3(3 / 2,3 / 3,3 /+)$, and in the presence of large opacity as Category 4 . The changes of predictive values for the forced expiratory volume in 1 second (FEV1), forced vital capacity (FVC), and $\mathrm{FEV}_{1} / \mathrm{FVC}$ ratio, and disability grade were evaluated using prediction equations. The results of pulmonary function test were categorized into no impairment (Fo: $\% \mathrm{FVC}$ and $\% \mathrm{FEV} 1 \geq 80 \%$ ), slight impairment (F1: 80\% > $\% \mathrm{FVC}$ or $\% \mathrm{FEV} 1 \geq 55 \%$ ), moderate impairment (F2: $55 \%$ $>\% \mathrm{FVC}$ or $\% \mathrm{FEV} 1 \geq 45 \%)$, and severe impairment $\left(\mathrm{F}_{3}\right.$ : $45 \%>\% \mathrm{FVC}$ or $\% \mathrm{FEV} 1$ ).

Information on gastric cancer was derived from the NHI database; patients with gastric cancer were defined as those with two or more claims of $\mathrm{C} 16$ malignant neoplasms of the stomach per KCD guideline as the main disease at inpatient stay and/or outpatient clinic visit between 2002 and 2017; for validation of the incidence of gastric cancer, a clearance period of 2 years was considered. Therefore, patients diagnosed with gastric cancer before 2004 and those without confirmed gastric cancer in 2016 and 2017 were excluded. Finally, the incidence of gastric cancer among the PHE participants was estimated from 1st January 2004 through 31 December 2015.

\section{Statistical analysis}

With regard to the risk of gastric cancers in our cohort, the age-standardized incidence ratio (SIR) and 95\% confidence intervals (CIs) were evaluated with adjustment of age into 5-year age groups and by indirect standardized method according to the following year. Structural data of the Korean population registered as residents in 2000 by the Ministry of the Interior and Safety of Korea and reported by Statistics Korea were considered to be that of the standard population. Cases with SIR and lower limit of $95 \%$ CI of $>1$ were considered to reflect a significantly higher risk of gastric cancer; the SIR value was obtained for each category of pneumoconiosis opacity. The relationship between risk of gastric cancer and lung function as an index of respiratory impairment, was investigated. In the assessment of the effect of dust type, results were compared between the coal workers group (mostly coal dust exposure) and the non-coal workers group (mostly silica exposure). In addition, attributable risk (AR) was calculated using the formula (SIR-1)/SIR. All analyses were conducted using SAS, version 9.4 (SAS Institute, Cary, NC, USA). A $p<0.05$ was considered to be statistically significant.

\section{Ethical consideration}

KCOMWEL and NHIS do not reveal information with the subject's personal identification to researchers, and therefore, the data in the present study are anonymized. The study was approved by the Institute Review Board (IRB) of Seoul St. Mary's Hospital (IRB approval number: KC18ZNSIo439). Written informed consent by the patients was waived due to a retrospective nature of our study.

\section{RESULTS}

A total of 151,536 of PHE records were obtained during the study period, which included multiple records for each participant; after acceptance of only the latest record in each, 47,498 were deemed eligible. Patients diagnosed with gastric cancer before 2004 and after $2015(\mathrm{n}=$ 2,766 ), and during a 10-year period after initial occupational dust exposure, which is within the limit of latency of cancer $(n=1,910)$, were excluded. Finally, a total of 42,822 participants were deemed as the eligible population (Fig. 1). Age distribution of gastric cancer in both study population and general population were shown in Supplementary Fig. 1.

The demographic and occupational characteristics of the study population are shown in Table 1. A total of 1,609 incidental cases of gastric cancer, derived from the 2004 to 2015 NHI database, was estimated among the PHE participants; the group with incidental gastric cancer was relatively older, predominantly of male sex, and more likely to have worked in mining industries than the group without gastric cancer.

The annual incidence rate of gastric cancer was 269.5 per 100,000 among workers with dust exposure versus 119.4 per 100,000 among the over 45 age group in the general population. The results of comparison of the incidence of gastric cancer between the PHE participants and general population are shown in Table 2. In 


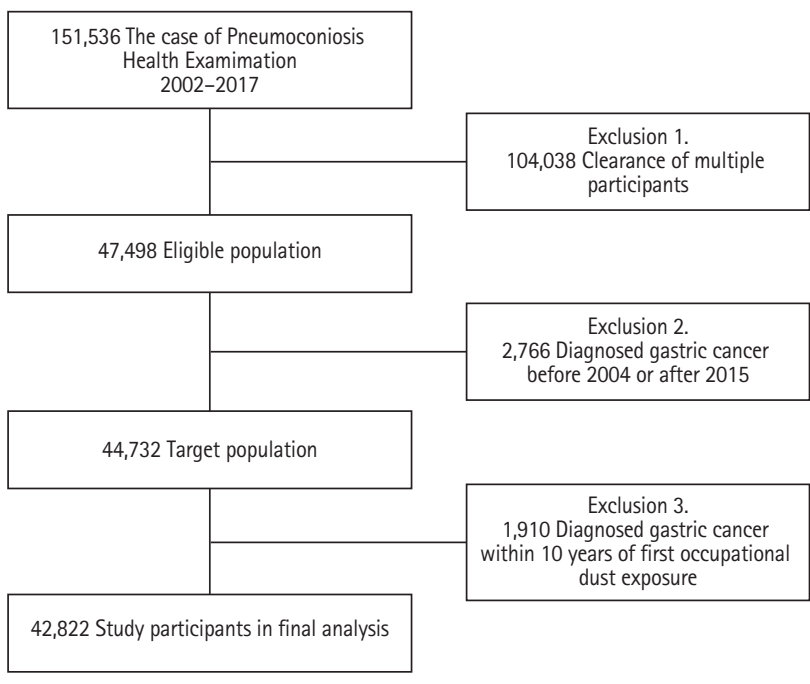

Figure 1. Schematic diagram of study participants.

the comparison with the reference group, SIR of gastric cancer was generally higher in male workers with dust exposure than in female workers. Among the male participants, the incidence of gastric cancer during 2004 to 2015 was 1,543 , while the expected value of that was 1,174 , which yielded SIR of 1.314 (95\% CI, 1.249 to 1.380 ); moreover, AR for dust exposure was $23.9 \%$ (95\% CI, 19.9\% to $27.5 \%)$.

In sensitivity analysis to determine whether men occupied in coal mining industry are predisposed to development of gastric cancer, higher SIR was achieved in male coal workers than in non-coal workers (SIR, 1.318 vs. 1.267). After categorizing male participants according to pneumoconiosis opacity and lung function, no clear dose-response relationship was observed with the increase of pneumoconiosis opacity, while the lung function categories were inversely related to the risk of gastric cancer ( $p$ for trend $=0.04$ ). In the no impairment group, SIR of gastric cancer was 1.741, whereas in the subgroup with severe impairment of lung function, no increase in the risk of gastric cancer were observed (Fig. 2).

\section{DISCUSSION}

In the present study, male workers who were exposed to dust attained a significantly higher incidence rate of gastric cancer than the rest of the population; we ob- tained an SIR value of 1.314 (95\% CI, 1.249 to 1.380) in those workers. After categorization of the group of male participants, there was no clear dose-response relationship between the increase of opacity and cancer risk; whereas, we observed a significant inverse correlation between the degree of lung function impairment and risk of gastric cancer.

Previous reports have indicated positive associations between the risk of gastric cancer and various occupational groups, particularly dusty occupations such as mining, construction, rubber manufacturing, metal processing, and carpentry [15-17]. Nevertheless, many of those studies reported results without statistical significance despite the large size of the samples $[18,19]$. Lee et al. [4] conducted a systematic review and meta-analysis of occupational exposure to crystalline silica and gastric cancer, and reported an effect size of 1.25 (95\% CI, 1.18 to 1.34) among 20 cohorts and nine case-control studies.

Dust such as crystalline silica and coal type may increase the risk for gastric cancer through several potential mechanistic pathways. First, workers could inhale carcinogens in their work environment, which may be swallowed and migrate into the stomach. Crystalline silica and other inflammatory agents cause irritation of the gastric mucosa and may increase the risk of gastric cancer $[18,20]$. After swallowing of the inhaled dust, the particles are introduced to the acidic environment of the stomach, where they may interact with nitrosating agents, such as nitrite [4]; at the cellular level, dust induces acute or chronic gastric inflammation, which could trigger gene mutation and increase the risk of chromosomal instability. Second, workers with intense dust exposure, especially miners, are usually exposed to a combination of different dust types including asbestos, metals like chromium and arsenic, radon, and diesel exhaust, all of which contain carcinogenic chemicals, such as polycyclic aromatic hydrocarbons. Third, reports indicate that differences in socioeconomic status, lifestyle, and susceptibility are the main causal factors of the increased risk of gastric cancer in workers with occupational dust exposure as compared to those in the general population [21]; workers who are exposed to dust usually belong to lower socioeconomic groups, and may consume unhealthy food, particularly high salt and low proportions of fruit and vegetable which are associated with increase in the risk for gastric cancer [22]. 
Table 1. General characteristics of study participants and incidence rate of gastric cancer during observation periods (2004 to 2015 )

\begin{tabular}{|c|c|c|c|c|}
\hline Variable & Total & Gastric cancer & Incidence rate, \% & $p$ value \\
\hline \multicolumn{5}{|l|}{ Age, yr } \\
\hline$\leq 44$ & $2,565(6.0)$ & $34(2.1)$ & 1.33 & $<0.01$ \\
\hline $45-49$ & $4,073(9.5)$ & $79(4.9)$ & 1.94 & \\
\hline $50-54$ & $6,285 \quad(14.7)$ & $161(10.0)$ & 2.56 & \\
\hline $55-59$ & $7,600 \quad(17 \cdot 7)$ & $290(17.9)$ & 3.82 & \\
\hline $60-64$ & $9,539(22.3)$ & $429(26.5)$ & 4.50 & \\
\hline $65-69$ & $7,613(17.8)$ & $391(24.2)$ & 5.14 & \\
\hline $70-74$ & $3,599(8.4)$ & $183(11.3)$ & 5.08 & \\
\hline $75-79$ & $1,120 \quad(2.6)$ & $37(2.3)$ & $3 \cdot 30$ & \\
\hline $8 o-84$ & $356(0.8)$ & $13(0.8)$ & 3.65 & \\
\hline$\geq 85$ & $72(0.2)$ & 1 (0.1) & 1.39 & \\
\hline Sex & & & & $<0.01$ \\
\hline Male & $40,115(93.7)$ & $1,577(97 \cdot 5)$ & 3.93 & \\
\hline Female & $2,707(6.3)$ & $41(2.5)$ & 1.51 & \\
\hline \multicolumn{5}{|l|}{ Working years $^{\mathrm{a}}$} \\
\hline $1-9$ & $25,059 \quad(60.3)$ & $961 \quad(61.2)$ & 3.83 & 0.30 \\
\hline $10-19$ & $11,297(27.2)$ & $437(27.9)$ & 3.87 & \\
\hline $20-29$ & $4,267 \quad(10.3)$ & $139(8.9)$ & 3.26 & \\
\hline$\geq 30$ & $906(2.2)$ & $32(2.0)$ & $3 \cdot 53$ & \\
\hline \multicolumn{5}{|c|}{ Category of radiologic finding ${ }^{\mathrm{b}}$} \\
\hline 0 & $21,485(52.2)$ & $787(50.1)$ & 3.66 & 0.60 \\
\hline $\mathrm{o} / 1$ & $3,156(7 \cdot 7)$ & $120(7 \cdot 6)$ & 3.80 & \\
\hline 1 & $9,427(22.9)$ & $398(25 \cdot 3)$ & 4.22 & \\
\hline 2 & $3,607(8.8)$ & $145(9.2)$ & 4.02 & \\
\hline 3 & $432(1.1)$ & $19(1.2)$ & 4.40 & \\
\hline 4 & $3,034(7 \cdot 4)$ & $102(6.5)$ & $3 \cdot 36$ & \\
\hline \multicolumn{5}{|c|}{ Results of pulmonary function test ${ }^{\mathrm{c}}$} \\
\hline Fo & $23,657(66.9)$ & $823(68.8)$ & 3.48 & 0.04 \\
\hline $\mathrm{F}_{1}$ & $9,017 \quad(25.5)$ & $300(25.1)$ & $3 \cdot 33$ & \\
\hline $\mathrm{F} 2$ & $1,470(4.2)$ & $47(3.9)$ & 3.20 & \\
\hline $\mathrm{F}_{3}$ & $1,224(3.5)$ & $27(2.3)$ & 2.21 & \\
\hline \multicolumn{5}{|c|}{ Occupational category $^{\mathrm{d}}$} \\
\hline Coal workers & $39,096(91.7)$ & $1,498(93.1)$ & 3.83 & 0.04 \\
\hline Non-coal workers & $3,527 \quad(8.3)$ & 111 (6.9) & 3.15 & \\
\hline Total & $42,822(100)$ & $1,609(100)$ & 3.76 & \\
\hline
\end{tabular}

${ }^{\mathrm{a}}$ Missing = 1,293.

${ }^{\mathrm{b}}$ Missing = 1,681.

${ }^{\mathrm{c}}$ Missing = 7,454.

${ }^{\mathrm{d}}$ Missing $=199$. 
Table 2. Age-SIR and AR of gastric cancer among dust-exposed workers.

\begin{tabular}{|c|c|c|c|c|}
\hline & Observed & Expected & $\operatorname{SIR}(95 \% \mathrm{CI})$ & $\operatorname{AR}(95 \% \mathrm{CI})$ \\
\hline \multicolumn{5}{|l|}{ According to sex } \\
\hline Female & 41 & $39 \cdot 3424$ & $1.0421(0.7239$ to 1.3604$)$ & $0.0404(-0.3815$ to 0.2649$)$ \\
\hline Male & 1,543 & $1,174.09$ & $1.3142(1.2488$ to 1.3797$)$ & $0.2391(0.1992$ to 0.2752$)$ \\
\hline \multicolumn{5}{|c|}{$\begin{array}{l}\text { According to category of radiologic } \\
\text { finding among male }\end{array}$} \\
\hline 0 & 733 & 504.438 & $1.4531(1.3481$ to 1.5581$)$ & $0.3118(0.2582$ to 0.3582$)$ \\
\hline $\mathrm{o} / 1$ & 114 & 93.6506 & $1.2173(0.9945$ to 1.4401$)$ & $0.1785(-0.0055$ to 0.3056$)$ \\
\hline 1 & 390 & 311.384 & $1.2525(1.1284$ to 1.3765$)$ & $0.2016(0.1138$ to 0.2735$)$ \\
\hline 2 & 143 & 117.899 & $1.2129(1.0147$ to 1.4112$)$ & 0.1755 (0.0144 to 0.2914$)$ \\
\hline 3 & 19 & $14 \cdot 5848$ & $1.3027(0.7212$ to 1.8843$)$ & $0.2324(-0.3866$ to 0.4693$)$ \\
\hline 4 & 100 & 102.163 & $0.9788(0.7875$ to 1.1702$)$ & $-0.0216(-0.2699$ to 0.1454$)$ \\
\hline \multicolumn{5}{|c|}{$\begin{array}{l}\text { According to results of pulmonary } \\
\text { function test among male }\end{array}$} \\
\hline Fo & 804 & 461.941 & $1.7405(1.6228$ to 1.8582$)$ & $0.4255(0.3838$ to 0.4618$)$ \\
\hline $\mathrm{F} 1$ & 293 & 198.347 & $1.4772(1.3113$ to 1.6431$)$ & 0.3231 (0.2374 to 0.3914$)$ \\
\hline $\mathrm{F} 2$ & 47 & 36.9623 & $1.2716(0.9147$ to 1.6285$)$ & $0.2136(-0.0933$ to 0.3859$)$ \\
\hline $\mathrm{F}_{3}$ & 27 & 28.8099 & $0.9372(0.5889$ to 1.2855$)$ & $-0.0670(-0.6981$ to 0.2200$)$ \\
\hline \multicolumn{5}{|c|}{$\begin{array}{l}\text { According to results of occupational } \\
\text { category among male }\end{array}$} \\
\hline Coal workers & 1,434 & $1,087.82$ & $1.3182(1.2501$ to 1.3863$)$ & $0.2414(0.2001$ to 0.2787$)$ \\
\hline Non-coal workers & 103 & 81.2711 & $1.2674(1.0233$ to 1.5115$)$ & $0.2110(0.0227$ to 0.3384$)$ \\
\hline
\end{tabular}

SIR, standardized incidence ratio; AR, attributable risk; CI, confidence interval.

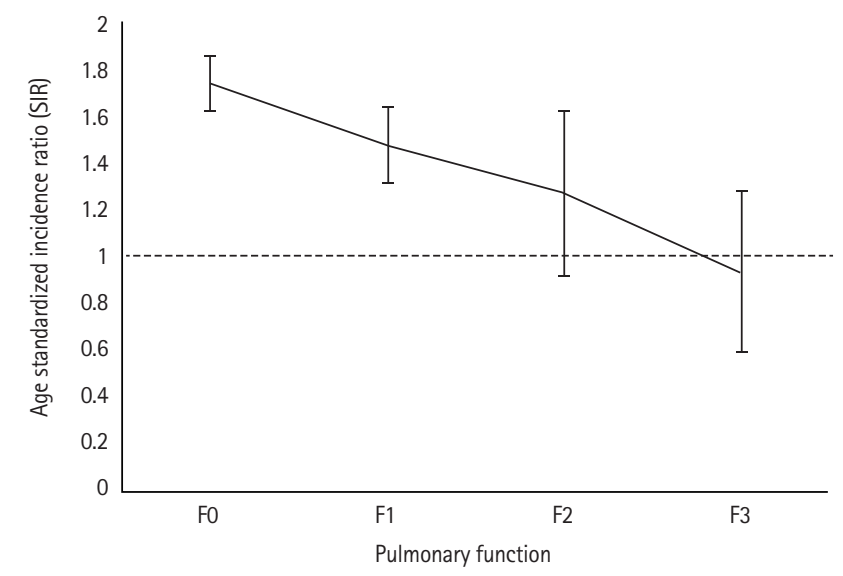

Figure 2. Age-standardized incidence ratio of gastric cancer according to respiratory impairment status.

A negative trend of association between the impairment of lung function and SIR of gastric cancer among workers with dust exposure is shown in Table 2. Functional impairment of the lung can prevent the inhaled dust from migrating to the digestive tract. Meyer et al. [23] proposed that people with impaired pulmonary clearance have a reduced risk of stomach cancer, based on the fact that the coal dust did not reach the stomach, but remained in the lungs; in contrast, those authors reported that the inhaled dust was readily transported into the digestive tract in workers with normal clearance mechanism. These findings are supported by another epidemiologic study investigating mortality in 3,790 coal miners in the Netherlands and role of the clearance mechanism; the results of that study indicated that the group with normal lung function achieved a higher level of SMR (1.54; 95\% CI, 1.26 to 1.87) than the group with impaired lung function (0.72; 95\% CI, 0.28 to 1.47) [21]. In agreement, we observed that in workers with dust exposure, impairment of the pulmonary clearance mechanism was associated with the decrease of SIR of gastric cancer. However, decreased lung function is not an indication for an endoscopy; hence, dust exposed workers 
with $\mathrm{F}_{2}$ or $\mathrm{F}_{3}$ grade might not participate in the gastric cancer screening. Further studies with adjustments for information bias regarding the eligibility for endoscopy among participants should be performed in the future.

In the present study, male coal workers attained a higher SIR (1.318) of gastric cancer than non-coal workers, and the variation in SIR could be due to the differences of dust exposure in each industry. A previous study on worker's environmental exposure in mining industries reported that the range of respirable dust concentration at various mines was 0.35 to $5.15 \mathrm{mg} / \mathrm{m}^{3}$ [24]; whereas, another study focused on exposure to respirable dust in non-mining dusty industries reported that the range of that in foundry, ceramics, stonemason, and other manufacturing industries was 0.97 to $1.35,1.06$ to $1.25,0.27$ to 1.38 , and 0.30 to $1.00 \mathrm{mg} / \mathrm{m}^{3}$, respectively [25]. The collective findings indicate that respirable dust levels are higher in mining industries than in nonmining industries, and consequently, the differences in exposure may be associated with the difference in SIR of gastric cancer between the workers in those two industry types.

Our study has several limitations. First, limited assessment of parameters due to restricted information on dust exposure may cause bias. The KCOMWEL database includes information of work history, medical examination history, and the exposure dust type, but not the exposure intensity. Moreover, records of working history by subcontractor before 1980 were incompletely registered in electronic database. Therefore, we could not exactly determine the cumulative dust exposure levels. In order to address this issue, KCOMWEL should first evaluate dust exposure of the PHE participants in the workplace; in this manner, subjects with no history of dust exposure would be excluded, and information bias may be reduced. Second, we assessed the diagnosis of gastric cancer according to the definition of KCD in the medical claims database without pathological confirmation through review of medical charts. NHI classifies Korean cancer patients as those with rare and intractable diseases (RID). A previous validation study with comparison between the NHI database and RID registry reported the sensitivity and specificity of disease of $97 \%$ to $98 \%$ and $93 \%$, respectively [26]. Third, adequate data of some potential confounders, including $H$. pylori infection and diet are lacking; such individual informa- tion would enable more robust statistical analysis. Some studies reported the $H$. pylori infection rates across occupations, but the results are inconsistent [27]. Moreover, while female and male individuals may attain similar rates of $H$. pylori infection, SIR of gastric cancer appears to be higher in male workers (usually underground workers) as compared to that in female workers (surface workers) in dusty industry, which reflects that dust may influence the mechanism of $\mathrm{H}$. pylori infection. Testing of this hypothesis exceeded the scope of the present study; nevertheless, in the future, we plan to conduct further analyses while controlling for the confounding effects of an $\mathrm{H}$. pylori infection. Fourth, we did not assess the screening of gastric cancer in the participants, and investigators should consider that such screening would allow early detection of gastric cancer; a study with further evaluation by gastric cancer screening among dust exposed workers s needed.

Despite these limitations, the present study raises important questions regarding the relationship between dust exposure and gastric cancer in Korea. With regard to the role of dusty work environments in the development of gastric cancer, we estimated that the proportion of cases attributable to such work was $23.9 \%$ among the male participants (95\% CI, 19.9 to 27.5 ) in our study. The results of subgroup analyses according to pulmonary function are relevant to clinical occupational medicine, and would enable better prevention of gastric cancer among workers with dust exposure. Although this study has provided evidence of relationship between dust exposure and gastric cancer, further research into the use of gastric cancer-related mortality would be of value.

In conclusion, dust-exposed workers showed a relatively higher incidence of gastric cancer than the general Korean population. Educational programs on scheduled gastric cancer screening and treatments for the eradication of $H$. pylori infection might lead to the early diagnosis, treatment, and prevention of gastric cancer among dust-exposed workers.

\section{KEY MESSAGE}

1. Dusty workers showed an increased incidence of gastric cancer. Nevertheless, registry and literature-based results do not sufficiently sup- 
port an association between dust exposure and risk of gastric cancer.

2. The present findings raise important questions regarding the relationship between dust exposure and gastric cancer. If dusty work environment is a significant causal factor in gastric cancer, our result that $23.9 \%$ of cases in the study population were attributable to such work, reflects the real life situation.

3. Lung function was inversely correlated with the risk of gastric cancer. Clinical studies including workers in dusty environments are needed to help prevent gastric cancer through early eradication of Helicobacter pylori infection as well as early diagnosis.

\section{Conflict of interest}

No potential conflict of interest relevant to this article was reported.

\section{Acknowledgments}

This work was supported by the Ministry of Employment and Labor and the Korea Workers' Compensation and Welfare Service.

\section{REFERENCES}

1. Lee SH, Kim DS, Kim YW, et al. Association between occupational dust exposure and prognosis of idiopathic pulmonary fibrosis: a Korean national survey. Chest 2015;147:465-474.

2. Hancock DG, Langley ME, Chia KL, Woodman RJ, Shanahan EM. Wood dust exposure and lung cancer risk: a meta-analysis. Occup Environ Med 2015;72:889-898.

3. Kim KH, Kabir E, Kabir S. A review on the human health impact of airborne particulate matter. Environ Int 2015;74:136-143.

4. Lee W, Ahn YS, Lee S, Song BM, Hong S, Yoon JH. Occupational exposure to crystalline silica and gastric cancer: a systematic review and meta-analysis. Occup Environ Med 2016;73:794-801.

5. Bray F, Ferlay J, Soerjomataram I, Siegel RL, Torre LA, Jemal A. Global cancer statistics 2018: GLOBOCAN estimates of incidence and mortality worldwide for 36 can- cers in 185 countries. CA Cancer J Clin 2018;68:394-424.

6. World Health Organization. Fact sheets: cancer [Internet]. Geneva: World Health Organization, c2018 [cited 2020 Apr 22]. Available from: https://www.who.int/news-room/ fact-sheets/detail/cancer.

7. Scheiman JM, Cutler AF. Helicobacter pylori and gastric cancer. Am J Med 1999;106:222-226.

8. Crew KD, Neugut AI. Epidemiology of gastric cancer. World J Gastroenterol 2006;12:354-362.

9. Kono S, Hirohata T. Nutrition and stomach cancer. Cancer Causes Control 1996;7:41-55.

10. Krstev S, Dosemeci M, Lissowska J, Chow WH, Zatonski W, Ward MH. Occupation and risk of stomach cancer in Poland. Occup Environ Med 2005;62:318-324.

11. Aragones N, Pollan M, Gustavsson P. Stomach cancer and occupation in Sweden: 1971-89. Occup Environ Med 2002;59:329-337.

12. Cocco P, Ward MH, Buiatti E. Occupational risk factors for gastric cancer: an overview. Epidemiol Rev 1996;18:218-234.

13. Kim L, Kim JA, Kim S. A guide for the utilization of Health Insurance Review and Assessment Service national patient samples. Epidemiol Health 2014;36:e2014008.

14. Shin DW, Cho B, Guallar E. Korean National Health Insurance database. JAMA Intern Med 2016;176:138.

15. Sjodahl K, Jansson C, Bergdahl IA, Adami J, Boffetta P, Lagergren J. Airborne exposures and risk of gastric cancer: $\mathrm{a}$ prospective cohort study. Int J Cancer 2007;120:2013-2018.

16. Finkelstein MM, Verma DK. Mortality among Ontario members of the International Union of Bricklayers and Allied Craftworkers. Am J Ind Med 2005;47:4-9.

17. Ji J, Hemminki K. Socio-economic and occupational risk factors for gastric cancer: a cohort study in Sweden. Eur J Cancer Prev 2006;15:391-397.

18. Coggon D, Barker DJ, Cole RB. Stomach cancer and work in dusty industries. Br J Ind Med 1990;47:298-301.

19. Lin S, Wang X, Yano E, et al. Exposure to chrysotile mining dust and digestive cancer mortality in a Chinese miner/miller cohort. Occup Environ Med 2014;71:323-328.

20. Schins RP. Mechanisms of genotoxicity of particles and fibers. Inhal Toxicol 2002;14:57-78.

21. Swaen GM, Meijers JM, Slangen JJ. Risk of gastric cancer in pneumoconiotic coal miners and the effect of respiratory impairment. Occup Environ Med 1995;52:606-610.

22. Steinmetz KA, Potter JD. Vegetables, fruit, and cancer prevention: a review. J Am Diet Assoc 1996;96:1027-1039. 
23. Meyer MB, Luk GD, Sotelo JM, Cohen BH, Menkes HA. Hypothesis: the role of the lung in stomach carcinogenesis. Am Rev Respir Dis 1980;121:887-892.

24. Shin YC, Choi BS, Lee BK, et al. Exposure assessment of air contaminants by type of mines in Korea: focused on carcinogenic substances. J Korean Soc Occup Environ Hyg 2002;12:95-105.

25. Kim YW, Phee YG, Kim HW, Kim HA. Dust concentration by $37 \mathrm{~mm}$ closed-face cassette, impactor and cyclone in manufacturing industries. J Korean Occup Health 2000;39:76-86.
26. Kim HJ, Hann HJ, Hong SN, et al. Incidence and natural course of inflammatory bowel disease in Korea, 20062012: a nationwide population-based study. Inflamm Bowel Dis 2015;21:623-630.

27. Sierra MS, Hastings EV, Fagan-Garcia K, Colquhoun A, Goodman KJ. Epidemiology, transmission and public health implications of Helicobacter pylori infection in Western countries. In: Mikloos BG, ed. Helicobacter Pylori: A Worldwide Perspective 2014. Oak Park (IL): Bentham Science Publishers, 2014:25-79. 


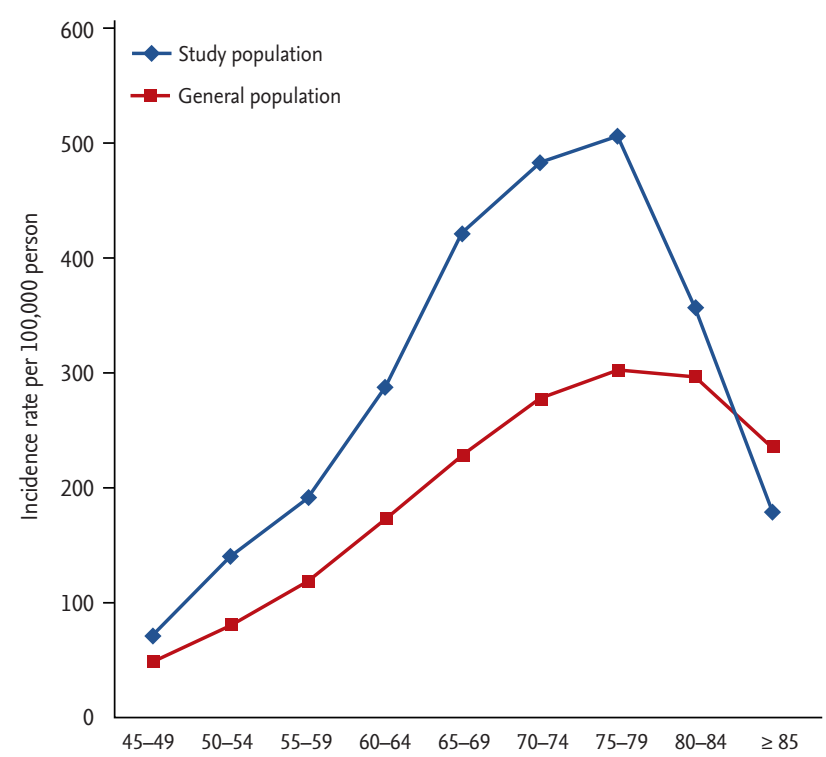

Supplementary Figure 1. Distribution of gastric cancer incidence rate (per 100,000 person). 